\title{
海南岛霸王岭热带雨林的种间分离
}

\author{
戴小华 余世孝* 练琚 $\square$ \\ (中山大学生命科学学院, 广州 510275)
}

摘 要 基于地理信息系统软件绘制了海南岛霸王岭热带雨林 1 个 $0.5 \mathrm{hm}^{2}$ 永久样地所有 $\mathrm{DBH} \geqslant 1 \mathrm{~cm}$ 树木的分布 图 进而借助最近邻体分析扩展模块判定每个个体的最近邻体植株, 并得到每个基株-最近邻体种对的距离。采用 $N \times N$ 最近邻体列联表的截表法, 研究了这个多物种群落的种间分离。结果表明, 随机毗邻种对占优势, 正分离种 对次之, 负分离种对最少。多数灌木和一些小乔木 (平均胸径 $<15 \mathrm{~cm}$ ) 彼此呈正分离, 少数负分离, 灌木和乔木间的 分离关系复杂 ;大乔木种类 (平均胸径 $\geqslant 65 \mathrm{~cm}$ ) 间不存在负分离。种间分离存在种间差异。种间分离与分布格局明 显相关 聚集分布的物种与其它物种正分离比例远比随机分布、均匀分布大:相反, 聚集分布物种与其它物种负分 离比例要比随机分布、均匀分布小。聚集聚集分布的种对最可能出现正分离; 聚集-均匀分布的种对以及随机-随 机分布的种对则最可能出现负分离。但是, 无论物种的分布格局或种对相对分布格局如何, 随机毗邻的种对都占 优势。与种间联结不同 种间分离反映的是小尺度的物种空间关系。在此基础上,初步阐述了种间分离的可能原 因及其生态学涵义。

关键词 种间分离 种间联结 $N \times N$ 最近邻体列联表 分布格局 地理信息系统

\section{INTERSPECIFIC SEGREGATION IN A TROPICAL RAIN FOREST AT BAWANGLING NATURE RESERVE , HAINAN ISLAND}

\author{
DAI Xiao-Hua YU Shi-Xiao* and LIAN Ju-Yu \\ ( School of Life Sciences, Zhongshan University , Guangzhou 510275 , China)
}

\begin{abstract}
Both interspecific association and interspecific segregation can be used to study the spatial affinity between different species. They are connected but different. Interspecific association is more related to habitat , while interspecific segregation more to small-scale effects and intra-/inter-specific interactions. Association is measured by plot sampling while segregation is measured by plotless nearest neighbor methods. Compared with interspecific association, few studies on pairwise segregation in a multi-species community have been reported. Meanwhile , clarifying the segregation relationship between different species pairs will be helpful for revealing the phenomena of the species interactions, community structure and community dynamics.

With the aid of GIS software (ArcView), a distribution map of all trees with $\mathrm{DBH} \geqslant 1 \mathrm{~cm}$ from a tropical rain forest community at Bawangling National Nature Reserve in Hainan Island was drawn. The nearest neighbors of each individual and the distances between every individual-neighbour pair were obtained by using ArcView's nearest-neighbor extension module (Jeff Jenness's Nearest Features v.3.5). Based on this, the spatial pattern and interspecific segregation in the multi-species community was studied with a subtable method of a $N \times N$ nearest-neighbor contingency table. Distribution pattern was measured by a revised Clark-Evans index $(C E)$ and the often-used $\chi^{2}$ method. Pielou's coefficient of segregation $(S)$ was adopted to measure the segregated degree between two species : while $0.7 \leqslant S \leqslant 1$, two species are positively segregated $;-1 \leqslant S \leqslant-0$. 7 , negatively segregated ; otherwise they are random neighbors. In order to solve zero cell problems, each zero cell of the contingency table had 0.001 added to it. This will not change the polarity of segregation and the zero denominators will be avoided in Pielou's equation. Pairwise segregation was shown in a constellation diagram in which solid lines stand for positive segregation , dash lines for negative segregation and different shapes for different mean DBH. The subtable method is quick, convenient and reliable. Its reliability increases with the increase of number of individuals. This is the reason we only focus on the segregation relationship between those species with more than 10 individuals. With the development of GIS software, computer simulation as well as the application of plotless sampling methods in a large area becomes possible. However, there is not any significant test for Pielou's coefficient until today. The statistical test for such subtables is still under question for both ecologists and statisticians.
\end{abstract}


In the forest community we analyzed, some species pairs are positively segregated $(23.3 \%)$ and a few pairs are negatively segregated $(2.4 \%)$. In contrast, the segregation relations between most of the species are random $(74.3 \%)$. Our results support Pielou's opinion that negative segregation is rare in a mature plant community. On the other hand, most understory trees (mean $\mathrm{DBH}<15 \mathrm{~cm}$ ) are positively segregated between each other, few negatively segregated. No negative segregation occurs between large arbors (mean $\mathrm{DBH} \geqslant 65$ $\mathrm{cm})$. Segregation relations between understory trees and large arbors are complicated. Meanwhile, most species will not be negatively segregated with others. Some will not be positively segregated with others. Two species have both positive and negative segregated relationship, while only one species has neither positive nor negative segregation with all other species. The results also indicated that interspecific segregation is closely related to their distribution patterns. Proportion of positive segregation between clumped species and other species $(31.4 \%)$ is much larger than that of random species $(16.7 \%)$ or uniform species $(20.0 \%)$. Proportion of negative segregation between clumped species and other species $(1.3 \%)$ is less than that of random $(3.3 \%)$ or uniform species $(2.4 \%)$. Clumped-clumped species pairs tend to be more positively segregated $(43.1 \%)$ than that of other species pairs. Both clumped-uniform and random-random species pairs are more likely to be negatively segregated, with a proportion of $5.6 \%$ and $4.8 \%$ respectively. Whatever the species distribution or the pairwise relative distribution is , the proportion of random neighbor pairs is the largest.

Possible causes and ecological meanings of interspecific segregation were also discussed in this paper. Interspecific segregation results from the combination of many biotic or abiotic factors. For example, negative segregation may occur between dependent unions or those species with similar habitat requirements. Intensive intraspecific competition may result in random or even uniform distribution , therefore such species are apt to be negatively segregated with other species. Large arbors tend to be positively segregated with other large arbors since their size prevents them from occupying a small space. Clumped species should have more conspecific neighbors, that is , less heterospecific neighbors. Thus they have some positive but no negative segregation relationships.

Key words Interspecific segregation, Interspecific association, $N \times N$ nearest-neighbor contingency table , Distribution pattern , GIS

种间分离 (Interspecific segregation)指的是种间个 体交错分布的程度, 它以两个物种个体的邻体关系 为基础 (Pielou，1961)。种间分离可以是正分离 (种 $\mathrm{A}$ 个体罕与种 $\mathrm{B}$ 个体毗邻) 、负分离 (种 $\mathrm{A}$ 个体常与 种 $\mathrm{B}$ 个体毗邻)或者两者都不是 (随机毗邻) (Dixon, 1994）。种间分离的研究对于揭示种间相互作用、群 落组成与动态具有重要意义。种间分离和种间联结 (Interspecific association)均可用于植物种间空间亲和 性的研究, 国内有关的研究主要集中在种间联结, 种 间分离则罕有报道。国外的研究则集中于两组个体 的分离如动植物种群 (Pielou,1961; Whipple,1980; Diggle ,1983)、母树-幼苗 ( Hamill \& Wright ,1986)、雌 株-雄株 (Bawa \& Opler ,1977)。

研究种间分离的方法主要有最近邻体列联表 (Pielou，1961)、最近邻体距离法 (Diggle,1983) 与 $K$ $(t)$ 方程 (Ripley,1981)。最近邻体列联表应用较为 广泛 (Dixon,1994) , 但只限于两物种群落。最近邻体 距离法则主要用于研究单种分布格局, $K(t)$ 方程适 用于多物种群落 (Coomes et al ，1999) , 目前应用最 为广泛, 但其涉及到复杂的 Monte Carlo 模拟。Reich 和 Davis(1998)介绍了一个适用于多物种群落的 $N$ $\times N$ 最近邻体列联表，用于分析草原优势种的总体
分离, 并未涉及具体种对的分离。

作为主要应用于景观层次的研究工具, 地理信 息系统(GIS) 技术在群落分析中的作用也日渐受到 重视(余世孝等，1998;2001a)。在以往的野外调查 中 如何判定所有个体的最近邻体及其距离是极其 费时费力的工作。Pielou (1961) 因此指出, 邻体分析 的样地应该足够小, 以便可以检查每个个体。在 GIS 软件以及最近邻体分析模块支持下，我们可以 方便、快捷地获得任何大小、任何形状区域内所有个 体的最近邻体及其距离。此外, 我们采用 $N \times N$ 最 近邻体列联表的截表方法, 可用于研究多物种的海 南岛霸王岭热带雨林中的种间分离规律。

\section{1 研究方法}

\section{1 研究地点与取样}

研究地点位于海南岛霸王岭国家级自然保护 区约 $18^{\circ} 50^{\prime} \sim 19^{\circ} 05^{\prime} \mathrm{N}, 109^{\circ} 05^{\prime} \sim 109^{\circ} 25^{\prime} \mathrm{E}$, 海拔 $700 \sim 1430 \mathrm{~m}$ 。气候为季风热带气候, 年平均温度 $23.6{ }^{\circ} \mathrm{C}$, 年均降水量 $1500 \sim 2000 \mathrm{~mm}$ 。土壤为山 地砖红壤。有关该研究区域的植被、区系研究近年 已有多篇报道 (余世孝等, 1993;2001b 练琚口等， 2001)。 
用于本文数据分析的为 2000 年建立的永久样 地，面积为 $50 \mathrm{~m} \times 100 \mathrm{~m}$,位于保护站旧址东北侧的 原始沟谷雨林, 坡向南向, 地形略有起伏, 露石多, 两 侧各有一条小河沟。群落高度为 $20 \sim 35 \mathrm{~m}$, 结构复 杂, 乔木上层主要优势种为重阳木 (Bischofia javani$c a$ )、笔管榕 (Ficus wightiana)、水翁 (Cleistocalyx operculatus)、布拉栎 (Quercus blakei)、海南梧桐（Firmiana hainanensis)、毛丹 (Phoebe hungmaoensis)、鸭脚木 (Schefflera octophylla)、黄叶树 (Xanthophyllum hainanense)、水石梓 (Sarcosperma laurinum) 等, 中下层主 要优势种为海南山龙眼 (Helicia hainanensis)、海南 倒吊笔（Wrightia hainanensis）、割舌罗（Walsura robusta)、大萼木姜（Litsea baviensis）、海南暗罗 (Polyalthia laui)、沙煲暗罗 ( P. consanguinea)、狗牙 花 (Ervatamia divaricata)、丛花灰木 ( Symplocos poilanei) 等。灌木层优势种有九节 (Psychortia rubra)、海南粗叶木 (Lasianthus hainanensis) 、鸡屎树 (L. cyanocarpos $)$ 等。草本层非常稀疏。

野外调查时, 将样地用样绳划分为 50 个 $10 \mathrm{~m} \times$ $10 \mathrm{~m}$ 的样方。记录每个样方内所有乔灌木 (胸径 $\mathrm{DBH} \geqslant 1 \mathrm{~cm}$ ) 的树号 (用铝牌标记)、种名、胸径、树 高、枝下高、冠幅、坐标 $(x, y)$ 。每个样方下角设 1 个 $1 \mathrm{~m} \times 1 \mathrm{~m}$ 小样方用于调查草本层。

借助 GIS 软件 (ArcView) 绘制出样地中 $\mathrm{DBH} \geqslant 1$ $\mathrm{cm}$ 的树木分布图, 比较每个植株与其附近所有个体 的距离来确定其最近邻体。首先固定的植株称为基 株, 距离的计算以及基株-最近邻体种对的判断, 由 ArcView GIS 软件扩展模块 Jeff Jenness' s Nearest Features v. 3.5 完成。

\section{2 分布格局测定}

Clark 和 Evans (1954) 的最近邻体法没有考虑到 样地的形状和边缘效应, 其检验向均匀分布倾斜。 Füldner(1995)提出了一个修正公式：

$$
C E=\frac{r_{A}}{r_{E}}=\frac{\frac{1}{N} \sum_{i=1}^{N} r_{i}}{0.5 \sqrt{A / N+0.0514 P / N+0.04 P / N^{3 / 2}}}
$$

式中 $C E$ 表示 Clark-Evans 指数; $r_{A}$ 为样地中每个个 体和其最近邻体间距离的平均值; $r_{E}$ 为样地中所有 个体随机分布时 $r_{A}$ 的预期值 ; $r_{i}$ 表示第 $i$ 个个体和 其最近邻体间的距离; $N$ 为样地内的个体总数; $A$ 为
样地面积; $P$ 为样地周长。当 $C E=1$ 时, 种群呈随 机分布, 当 $C E>1$ 时为均匀分布, 当 $C E<1$ 时为聚 集分布。实测与预测的偏离程度可用正态分布检验 (Kint et al. 2000) :

$$
u=\frac{r_{A}-r_{E}}{\sigma_{r_{E}}}
$$

式中

$$
\sigma_{r_{E}}=\frac{0.26136}{\sqrt{\rho N}}=\frac{0.26136}{\sqrt{N^{2} / A}}
$$

$r_{A}, r_{E}, N, A$ 同公式(1); $\rho=N / A$ 为样地中个体密 度 $; \sigma_{r_{E}}$ 为一个密度为 $\rho$ 符合 Poisson 分布的森林中 $r_{E}$ 的标准差。

为了比较无样地法和样地法的结果，我们也采 用了样地法常用的 $\chi^{2}$ 检验方法(王伯荪等, 1996)。 利用 $d$ 值来判断分布类型:

$$
d=\sqrt{2 \chi^{2}}-\sqrt{2 v-1}
$$

其中, $d$ 为标准正态变量, $v$ 是 $\chi^{2}$ 检验的自由度。 当 $d>1.960$ 时，表示聚集分布; $d<-1.960$ 时，表 示均匀分布; 否则, 种群为随机分布 (Wratten \& Fry， 1980)。

\section{3 种间分离测定}

de Jong 等 (1983) 采用了接触取样 (Contact sampling)种对的 $N \times N$ 列联表及其截取方法 (Subtable method），用于草本植被的联结分析。类似地，Reich 和 Davis (1998) 介绍了用于计算多物种群落总体分 离的 $N \times N$ 最近邻体列联表, 但基株是随机选取 的, 并非全体取样。而 Pielou (1961) 的 $2 \times 2$ 最近邻 体列联表中, 基株涵盖了群落中所有个体。对多物 种群落进行全体取样, 我们得到一个 $N \times N$ 最近邻 体列联表 (表 1 ) ,它实际上就是 Pielou 的 $2 \times 2$ 最近 邻体列联表的扩展。

截取上述 $N \times N$ 列联表得到关于种 $i$ 和种 $j$ 的 $2 \times 2$ 列联表 $($ 表 2$)$ 。

关于 $2 \times 2$ 截表的显著性检验存在争论。de Jong 等 (1983) 在接触取样中应用了这种截取方法 (Short-cut method) ,并认为普通的 $\chi^{2}$ 检验是有效的。 但 Reich 和 Davis (1998) 认为,这些截表 (Subtable) 并 非相互独立，因此 $\chi^{2}$ 检验既不适用，也没有必要。 因此，我们采用 Pielou (1961) 的分离指数 (Coefficient of segregation) 来计算种间的分离程度 :

$$
S=1-\frac{N_{i j}\left(n_{i j}+n_{j i}\right)}{\left(n_{i i}+n_{i j}\right)\left(n_{i j}+n_{j j}\right)+\left(n_{j i}+n_{j j}\right)\left(n_{i i}+n_{j i}\right)}=\frac{2\left(n_{i i} n_{i j}-n_{i j} n_{j i}\right)}{\left(n_{i i}+n_{i j}\right)\left(n_{i j}+n_{j j}\right)+\left(n_{j i}+n_{j j}\right)\left(n_{i i}+n_{j i}\right)}
$$


如果 $n_{i j}$ 值为 0 , 即意味着这类种对出现的概率很小, 我们给这些 $n_{i j}$ 加权 0.001 。其原因有三 其一, 不会 改变种间分离的性质; 其二, 避免公式 (3)中出现零 分母, 最重要的是, 多零值截表变得可以计算, 效果 很好, 也更符合实际。这种加权方法在种间联结列 联表中也经常使用 (王伯荪等, 1996)。分离指数 $S$ 的值变化于 -1 和 +1 之间, 当 $n_{i i}=n_{i j}=0$ 并且 $n_{i j}$ $=n_{j i} \neq 0$ 时，也就是说不存在同种毗邻时, $S$ 达到最 小值 -1 , 两个物种发生最大可能的正分离; 当 $n_{i j}=$ $n_{i i}=0$ 并且 $n_{i i}=n_{i j} \neq 0$ 时，也就是说两个物种不存 在相互毗邻时, $S$ 达到最小值 - 1 , 两个物种发生最 大可能的负分离; 当 $n_{i i} n_{i j}=n_{i j} n_{j i}$ 时, $S=0$, 两个物种 完全随机毗邻。但 Pielou 并未给出 $S$ 的统计检验方 法 (Reich \& Davis, 1998) , 我们在研究过程中也尝试 了 $\chi^{2}$ 检验和 $G$ 检验, 综合这些检验结果并在 ArcView软件上考察 $S$ 取不同值时的物种分离情况 后，我们规定，当 $0.7 \leqslant S \leqslant 1$ 时两个物种为正分离, 当 $-1 \leqslant S \leqslant-0.7$ 时为负分离即两个物种倾向于彼 此为邻, 当 $-0.7<S<0.7$ 时为既非正分离也非负
分离的随机毗邻种对。截表的显著性检验显然可以 更好地反映分离的性质与程度，但尚未找到其统计 理论基础。在没有找到合适的显著性检验方法之 前, 分离指数是个折衷的方法, 其区间划分多少有些 主观, 需要根据实际情况确立。

星座图用于表示各种对间的分离指数。用实线 代表正分离, 虚线代表负分离,并不表示距离;如果 没有实线或者虚线，则表示随机毗邻。同时用不同 形状代表植物的平均胸径大小, 用于了解不同径级 植物(灌木、小乔木、大乔木)之间的分离情况, 也可 以表达各类分离的状况。

\section{2 实验结果}

\section{1 分布格局}

由表 1 可见 最近邻体法的结果是 该热带雨林 41 个种群中, 有 21 个呈随机分布, 18 个呈聚集分 布, 仅 2 个呈均匀分布。 $\chi^{2}$ 检验的结果是: 24 个种 群呈随机分布, 17 个呈聚集分布, 没有均匀分布。 两种方法的结果相当一致。

表 $1 N \times N$ 最近邻体列联表

Table $1 \quad N \times N$ nearest-neighbor contingency table

\begin{tabular}{|c|c|c|c|c|c|c|}
\hline \multirow{2}{*}{$\begin{array}{c}\text { 基株 } \\
\text { Base plant }\end{array}$} & \multicolumn{6}{|c|}{ 最近邻体 Nearest neighbor } \\
\hline & 种 $1 S_{1}$ & 种 $2 S_{2}$ & 种 $3 S_{3}$ & $\cdots$ & 种 $k S_{k}$ & 总计 Total \\
\hline 种 $1 S_{1}$ & $n_{11}$ & $n_{12}$ & $n_{13}$ & $\cdots$ & $n_{1 \mathrm{k}}$ & $f_{1}$ \\
\hline 种 $2 S_{2}$ & $n_{21}$ & $n_{22}$ & $n_{23}$ & $\cdots$ & $n_{2 \mathrm{k}}$ & $\mathrm{f}_{2}$ \\
\hline 种 $3 S_{3}$ & $n_{31}$ & $n_{32}$ & $n_{33}$ & $\cdots$ & $n_{3 \mathrm{k}}$ & $\mathrm{f}_{3}$ \\
\hline$\ldots$ & $\ldots$ & $\ldots$ & $\ldots$ & $\ldots$ & $\ldots$ & $\cdots$ \\
\hline 种 $k S_{k}$ & $n_{k 1}$ & $n_{k 2}$ & $n_{k 3}$ & $\cdots$ & $n_{k k}$ & $\mathrm{f}_{k}$ \\
\hline 总计 Total & $s_{1}$ & $s_{2}$ & $s_{3}$ & $\cdots$ & $s_{\mathrm{k}}$ & $N$ \\
\hline
\end{tabular}

$S_{i}$ 种 $i$ Species $i \quad k$ 样地中总物种数 The number of species in the plot $n_{i j}$ 种 $i$ 个体的最近邻体是种 $j$ 的个体时的数目 Individuals of species $i$ whose nearest neighbor is an individual of species $j \quad N$ 样方内所有个体的总和 Number of all the individuals $f_{i}$ : 种 $i$ 的个体数 Individuals number of species $i \quad s_{i}$ : 以种 $i$ 为最近邻体的个体总数 The individuals number of species $i$ as nearest neighbor plants

表 $22 \times 2$ 最近邻体列联表

Table $22 \times 2$ nearest-neighbor contingency table

\begin{tabular}{clll}
\hline 基株 & \multicolumn{3}{c}{ 最近邻体植物 Nearest neighbor } \\
\cline { 2 - 4 } Base plant & 种 Species $S_{i}$ & 种 Species $S_{j}$ & 总计 Total \\
\hline 种 Species $S_{i}$ & $n_{i i}$ & $n_{i j}$ & $n_{i i}+n_{i j}$ \\
种 Species $S_{j}$ & $n_{j i}$ & $n_{j j}$ & $n_{j i}+n_{j j}$ \\
总计 Total & $n_{i i}+n_{j i}$ & $n_{i j}+n_{i j}$ & $N_{i j}$ \\
\hline
\end{tabular}

$N_{i j}$ 种 $i$ 和种 $j$ 个体之和, 其余符号同表 1 Total number of both species $i$ and species $j$, the other symbols same as Table 1

\section{2 种间分离}

我们研究了样地中所有株数大于 10 的树种 共 计 41 个物种, 820 组种对。研究表明, 该群落中随 机毗邻种对占大多数 $(74.3 \%)$,正分离种对有一些 $(23.3 \%)$ ，负分离种对很少 $(2.4 \%)$ (表 4$)$ 。图 1 为 该群落种间分离的星座图。大多数灌木和一些小乔 木 (平均胸径 $<15 \mathrm{~cm}$ ) 彼此呈正分离, 少数负分离。
灌木和乔木间的分离关系则较为复杂。大乔木种类 (平均胸径 $\geqslant 65 \mathrm{~cm}$ ) 间不存在负分离。由图 1 可见， 多数物种如九节不与其它物种形成负分离, 有些物 种如岭南山竹子 (Garcinia oblongifolia) 不与其它物种 发生正分离, 两个物种 (海南山龙眼和短药蒲桃 Syzygium brachyantherum) 则正、负分离均存在，只有 一个物种 (罗伞 Ardisia quinquegona) 仅与其它物种随 机毗邻。图 2 为样地中负分离、随机毗邻以及正分 离种对的典型例子。负分离的两个种如海南山龙眼 与黄叶树, 毗邻另一种的个体远较毗邻同种的多; 正 分离的种对如沙煲暗罗与卷边罗金 (Ardisia crena$t a)$,毗邻另一种的个体较毗邻同种的少; ;迶机毗邻 种对如海南暗罗与九节, 其毗邻关系是不定的。 
表 3 海南霸王岭热带雨林 41 个种群的分布格局

Table 3 Distribution patterns of 41 species in a tropical rain forest at Bawangling , Hainan

\begin{tabular}{|c|c|c|c|c|c|c|c|}
\hline \multirow{2}{*}{$\begin{array}{l}\text { 序号 } \\
\text { No. }\end{array}$} & \multirow[b]{2}{*}{$\begin{array}{l}\text { 物种 } \\
\text { Species }\end{array}$} & \multicolumn{3}{|c|}{ 最近邻体法 Nearest-neighbor method } & \multicolumn{3}{|c|}{$\chi^{2}$ 检验 Chi-square test } \\
\hline & & $C E$ & $\begin{array}{c}u \text { 值 } \\
u \text { value }\end{array}$ & $\begin{array}{c}\text { 分布格局 } \\
\text { Pattern }\end{array}$ & $\begin{array}{c}\chi^{2} \text { 值 } \\
\chi^{2} \text { value }\end{array}$ & $\begin{array}{c}d \text { 值 } \\
d \text { value }\end{array}$ & $\begin{array}{c}\text { 分布格局 } \\
\text { Pattern }\end{array}$ \\
\hline 1 & 九节 Psychotria rubra & 0.657 & $-9.515^{*}$ & $\mathrm{C}$ & $81.788^{*}$ & 9.184 & C \\
\hline 2 & 沙煲暗罗 Polyalthia consanguinea & 0.768 & $-4.884^{*}$ & $\mathrm{C}$ & $19.049^{*}$ & 3.527 & $\mathrm{C}$ \\
\hline 3 & 海南山龙眼 Helicia hainanensis & 0.984 & -0.290 & $\mathrm{R}$ & 3.060 & 0.238 & $\mathrm{R}$ \\
\hline 4 & 狗牙花 Ervatamia divaricata & 0.949 & -0.869 & $\mathrm{R}$ & 6.345 & 1.326 & $\mathrm{R}$ \\
\hline 5 & 海南倒吊笔 Wrightia hainanensis & 0.826 & $-2.712^{*}$ & $\mathrm{C}$ & $10.854^{*}$ & 2.423 & C \\
\hline 6 & 海南暗罗 Polyalthia laui & 0.832 & $-2.582^{*}$ & $\mathrm{C}$ & 4.866 & 0.884 & $\mathrm{R}$ \\
\hline 7 & 海南粗叶木 Lasianthus hainanensis & 0.844 & $-2.297^{*}$ & C & $14.470^{*}$ & 3.144 & C \\
\hline 8 & 丛花灰木 Symplocos poilanei & 0.866 & -1.861 & $\mathrm{R}$ & $22.771^{*}$ & 4.512 & C \\
\hline 9 & 割舌罗 Walsura robusta & 1.167 & $2.175^{*}$ & $\mathrm{U}$ & 1.311 & -0.113 & $\mathrm{R}$ \\
\hline 10 & 鸡屎树 Lasianthus cyanocarpos & 1.053 & 0.677 & $\mathrm{R}$ & 1.970 & -0.251 & $\mathrm{R}$ \\
\hline 11 & 卷边罗伞 Ardisia crenata & 0.919 & -0.989 & $\mathrm{R}$ & 5.693 & 1.138 & $\mathrm{R}$ \\
\hline 12 & 大营木姜 Litsea baviensis & 0.947 & -0.610 & $\mathrm{R}$ & 0.591 & 0.087 & $\mathrm{R}$ \\
\hline 13 & 黄叶树 Xanthophyllum hainanense & 1.116 & 1.347 & $\mathrm{R}$ & 0.145 & -1.194 & $\mathrm{R}$ \\
\hline 14 & 赛木患 Aphania oligophylla & 0.848 & -1.764 & $\mathrm{R}$ & 3.030 & 0.730 & $\mathrm{R}$ \\
\hline 15 & 野茶 Camellia sinensis var. assamica & 0.728 & $-3.063^{*}$ & C & $9.039^{*}$ & 2.016 & C \\
\hline 16 & 布渣叶 Microcos paniculata & 0.836 & -1.812 & $\mathrm{R}$ & $15.940^{*}$ & 3.410 & C \\
\hline 17 & 红果口木 Dysoxylum binectariferum & 1.107 & 1.184 & $\mathrm{R}$ & 2.887 & 0.671 & $\mathrm{R}$ \\
\hline 18 & 水翁 Cleistocalyx operculatus & 0.963 & -0.392 & $\mathrm{R}$ & 1.116 & 0.494 & $\mathrm{R}$ \\
\hline 19 & 柏拉木 Blastus cochinchinensis & 0.132 & $-8.804^{*}$ & $\mathrm{C}$ & $248.666^{*}$ & 20.065 & C \\
\hline 20 & 岭南山竹子 Garcinia oblongifolia & 0.788 & $-1.973^{*}$ & C & 1.275 & 0.597 & $\mathrm{R}$ \\
\hline 21 & 鸭脚木 Schefflera octophylla & 0.764 & $-2.200^{*}$ & $\mathrm{C}$ & $1767.681^{*}$ & 58.459 & C \\
\hline 22 & 短药蒲桃 Syzygium brachyantherum & 1.104 & 0.950 & $\mathrm{R}$ & 0.218 & -1.072 & $\mathrm{R}$ \\
\hline 23 & 谷木 Memecylon ligustrifolium & 0.592 & $-3.717^{*}$ & $\mathrm{C}$ & $48.889^{*}$ & 8.888 & C \\
\hline 24 & 三角瓣花 Prismatomeris tetranda & 0.593 & $-3.711^{*}$ & $\mathrm{C}$ & $10.313^{*}$ & 2.810 & C \\
\hline 25 & 水石梓 Sarcosperma laurinum & 0.971 & -0.262 & $\mathrm{R}$ & 1.895 & 0.947 & $\mathrm{R}$ \\
\hline 26 & 白木香 Aquilaria sinensis & 0.965 & -0.308 & $\mathrm{R}$ & 4.508 & 1.271 & $\mathrm{R}$ \\
\hline 27 & 尖峰润楠 Machilus monticola & 0.668 & $-2.952^{*}$ & $\mathrm{C}$ & $7.760^{*}$ & 2.207 & C \\
\hline 28 & 毛丹 Phoebe hungmaoensis & 1.112 & 0.992 & $\mathrm{R}$ & 1.309 & 0.618 & $\mathrm{R}$ \\
\hline 29 & 红紫麻 Oreocnide rubescens & 0.516 & $-4.071^{*}$ & C & $7.648^{*}$ & 2.911 & C \\
\hline 30 & 润楠 Machilus sp. & 0.867 & -1.123 & $\mathrm{R}$ & 1.717 & 0.853 & $\mathrm{R}$ \\
\hline 31 & 狭叶泡花 Meliosma angustifolia & 0.762 & $-2.004^{*}$ & $\mathrm{C}$ & 1.717 & 0.853 & $\mathrm{R}$ \\
\hline 32 & 红翅械 Acer fabri & 0.825 & -1.430 & $\mathrm{R}$ & 0.215 & -0.344 & $\mathrm{R}$ \\
\hline 33 & 长柄梭椤 Reevesia longipetiolata & 1.091 & 0.721 & $\mathrm{R}$ & 0.174 & -0.410 & $\mathrm{R}$ \\
\hline 34 & 乌柿 Diospyros cathayensis & 0.748 & $-1.994^{*}$ & C & 0.705 & 0.187 & $\mathrm{R}$ \\
\hline 35 & 高地山香园 Turpinia montana & 0.593 & $-3.115^{*}$ & $\mathrm{C}$ & $19.409^{*}$ & 5.230 & $\mathrm{C}$ \\
\hline 36 & 钝叶紫金牛 Ardisia obtuse & 0.969 & -0.226 & $\mathrm{R}$ & $12.211^{*}$ & 3.942 & C \\
\hline 37 & 罗伞 Ardisia quinquegona & 0.883 & -0.861 & $\mathrm{R}$ & 1.874 & 0.936 & $\mathrm{R}$ \\
\hline 38 & 乌营 Syzygium chunianum & 1.308 & $2.273^{*}$ & $\mathrm{U}$ & 0.005 & -0.900 & $\mathrm{R}$ \\
\hline 39 & 水同木 Ficus harlandii & 0.721 & $-1.982^{*}$ & C & $74.641^{*}$ & 11.218 & C \\
\hline 40 & 铁罗 Aglaia tsangii & 0.838 & -1.152 & $\mathrm{R}$ & $16.575^{*}$ & 4.758 & $\mathrm{C}$ \\
\hline 41 & 荔枝 Litchi chinensis var. euspontanea & 0.712 & $-2.047^{*}$ & $\mathrm{C}$ & 0.045 & -0.700 & $\mathrm{R}$ \\
\hline
\end{tabular}

* : p $<0.05 \mathrm{R}$ 随机分布 Random U 均匀分布 Uniform C 聚集分布 Clumped

\section{表 4 各类分布格局的物种与其它物种形成不同类型分离种对的比例}

Table 4 Proportion for various kinds of segregation between the species with a specific distribution pattern and other species (\%)

\begin{tabular}{lccc}
\hline $\begin{array}{c}\text { 分布格局 } \\
\text { Distribution pattern }\end{array}$ & $\begin{array}{c}\text { 正分离 } \\
\text { Positive segregation }\end{array}$ & $\begin{array}{c}\text { 随机毗邻 } \\
\text { Random neighbor }\end{array}$ & $\begin{array}{c}\text { 负分离 } \\
\text { Negative segregation }\end{array}$ \\
\hline 聚集分布物种 Clumped species & 31.4 & 67.4 & 1.2 \\
随机分布物种 Random species & 16.7 & 80.0 & 3.3 \\
均匀分布物种 Uniform species & 20.0 & 76.3 & 3.7 \\
总计 Total & 23.3 & 74.3 & 2.4 \\
\hline
\end{tabular}




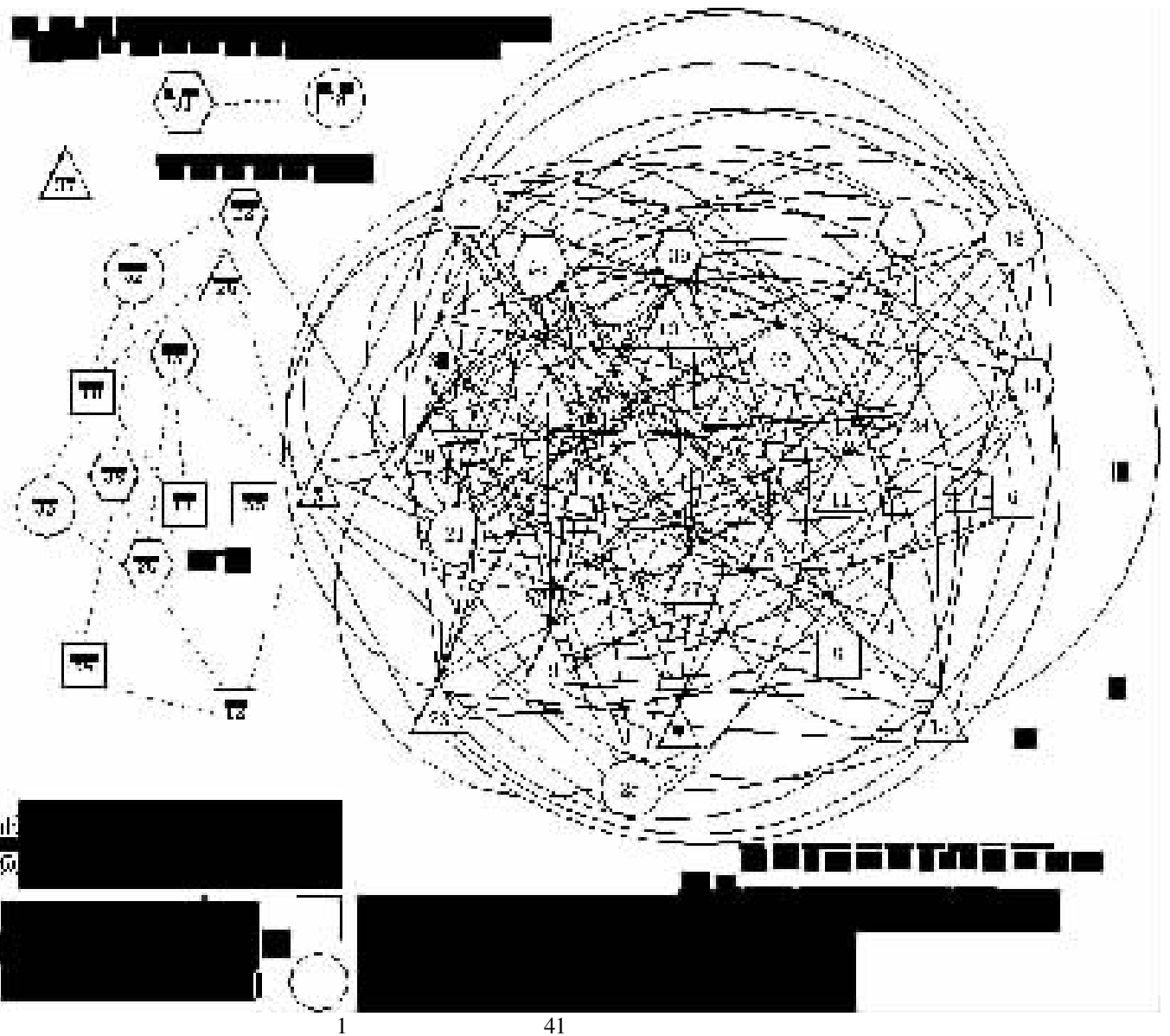

Fig. 1 Constellation diagrams of interspecific segregation of 41 species with more than 10 individuals in a ravine rain forest stand at Bawangling 种号同表 3 Legends to species refer to Table 3

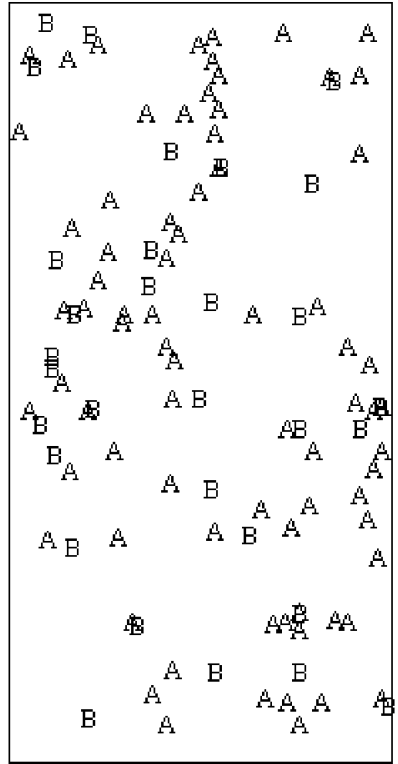

a
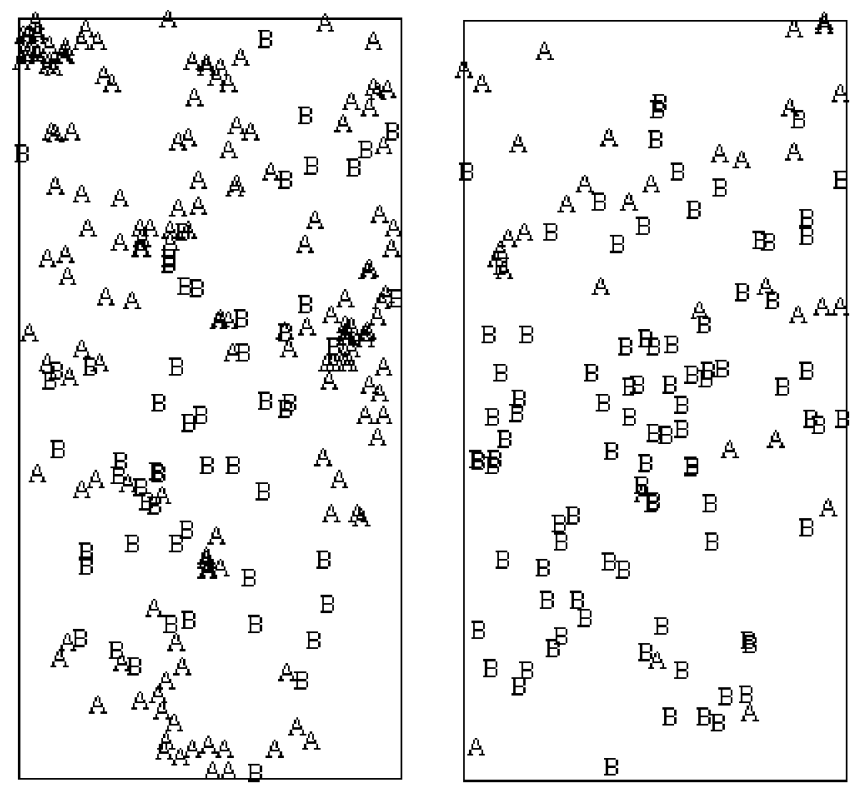

图 2 典型的种间分离格局

Fig.2 Typical patterns of interspecific segregation

$\mathrm{a}$ : 负分离种对 Negative segregated pair $S=-0.800$ (A 海南山龙眼 Helicia hainanensis B : 黄叶树 Xanthophyllum hainanense) b : 随机毗邻种 对 Random neighbor pair $S=0.496$ (A 节 Psychotria rubra B 海南暗罗 Polyalthia laui) c : 正分离种对 Positive segregated pair $S=0.815$ (A： 卷边罗伞 Ardisia crenata B 沙煲暗罗 Polyalthia consanguinea) 


\section{3 种间分离与分布格局}

由于种间分离采用的也是无样地的最近邻体方 法，因此本文分布格局采用 Clark-Evans 最近邻体法 的结果。表 4 表明, 聚集分布的物种与其它物种正 分离比例 $(31.4 \%)$ 远比随机分布 $(16.7 \%)$ 、均匀分 布 $(20.0 \%)$ 大 相反 聚集分布物种与其它物种负分 离比例 $(1.3 \%)$ 要比随机分布 $(3.3 \%)$ 、均匀分布
$(2.4 \%)$ 少。表 5 表明 种间分离与种对的相对分布 格局之间的关系也极其明显。均为聚集分布的种对 出现正分离的比例最大 $(43.1 \%)$, 但不会形成负分 离; 一为聚集另一为均匀分布的种对, 以及均为随机 分布的种对则最可能出现负分离，比例分别为 $5.6 \%$ 和 $4.8 \%$ 。但是, 无论物种的分布格局或种对相对 分布格局如何, 随机毗邻分布的种对都占优势。

表 5 各种分布格局组合的种对的分离状况

Table 5 Proportion for various kinds of segregation between the species pairs with different types of distribution pattern (\%)

\begin{tabular}{cccc}
\hline $\begin{array}{c}\text { 种对的分布格局 } \\
\text { Distribution pattern of species pair }\end{array}$ & $\begin{array}{c}\text { 正分离 } \\
\text { Positive segregation }\end{array}$ & $\begin{array}{c}\text { 随机毗邻 } \\
\text { Random neighbor }\end{array}$ & $\begin{array}{c}\text { 负分离 } \\
\text { Negative segregation }\end{array}$ \\
\hline 聚集-聚集 Clumped-Clump pair & 43.1 & 56.9 & 0 \\
聚集-随机 Clumped-Random pair & 23.5 & 74.6 & 1.9 \\
聚集-均匀 Clumped-Uniform pair & 11.1 & 83.3 & 5.6 \\
随机-随机 Random-Random pair & 13.8 & 81.4 & 4.8 \\
随机-均匀 Random-Uniform pair & 7.1 & 90.5 & 2.4 \\
均匀-均匀 Uniform-Uniform pair & 0 & 100 & 0 \\
总计 Total & 23.3 & 74.3 & 2.4 \\
\hline
\end{tabular}

\section{3 讨论与结论}

种间分离和种间联结均可用于研究两个种的联 合格局, 反映物种在空间上相互吸引或者排斥的性 质 两者既有联系又有区别。种间联结更多地与生 境关联，它的测定以样方为基础，受样方大小和间隔 的影响很大(Pielou,1969)。种间分离的测定以距离 为基础，不受样方大小、间隔甚至形状的影响。种间 分离与种间、种内相互作用密切相关, 是一个物种相 对于另一物种的格局 (Pielou,1969)，容易受到小尺 度效应的影响，反映了两个物种相互混杂( Intermingle) 的程度 (Greig-Smith , 1983)。Pielou(1961) 认为相 互混杂的程度是两物种联合种群的独立于尺度的内 在特性。种对相互混杂的程度越小, 种间分离的程 度就越大。如果两个种是随机混合的, 我们认为它 们不分离 (Non-segregation); 如果两个物种倾向于独 立成丛,同种混杂远多于异种混杂, 种对发生正分 离, 如果它们倾向于彼此混杂, 异种混杂远多于同种 混杂 那么种对发生负分离。

群落演替的早期, 可能会出现较多的负分离或 者正分离。负分离可能是由于不同植物的种子散播 在一起而成丛, 正分离则可能是因为生境异质性或 者种子散播在母树附近而导致同种植物聚集分布。 随着群落的发展, 竞争以及不适生境导致树木自然 稀疏, 是分离减少的重要原因。如果稀疏发生在单 种丛, 正分离将减少; 如果发生于异种丛, 负分离将
减少(Pielou,1969)。到了成熟群落阶段, 种间关系 已趋于稳定, 负分离可能出现, 但会很罕见(Pielou, 1977)。海南岛霸王岭热带雨林中, 随机毗邻种对占 优势, 正分离种对较少, 负分离种对很少。

种间分离是多种因素联合作用的结果。这些因 素包括定居者的初始分布、干扰历史、无性系生长或 种子散布的格局、非生物环境的异质性 ( Law et al. , 1993 )、自然稀疏、种间与种内相互作用 (竞争、他感、 捕食等)、植物形态特化、生境需求以及个体大小等， 它与分布格局反映的都是空间关系。Coomes (1999) 指出种间分离与种内聚集程度有关。物种聚集分布 说明其倾向于以同种个体为邻, 排斥异种个体, 所以 正分离情况较多, 负分离则未发现。相反, 如果种内 竞争激烈而导致物种随机分布甚至均匀分布, 那么 就易于出现负分离的情况。本研究结果支持 Coomes 的观点聚集分布的物种与其它物种正分离 的概率更大随机分布或均匀分布的物种比聚集分 布的物种更倾向于与其它物种负分离。聚集-聚集 分布的种对出现正分离的概率最大，但不会形成负 分离聚集-均匀分布的种对，以及随机-随机分布的 种对则最可能出现负分离。

研究结果也表明, 许多具有相近生境需求的物 种如林下灌木或顶层乔木存在负分离。一些灌木与 中上层乔木形成负分离, 可能与这些灌木耐荫习性 有关。研究也发现, 大乔木之间易发生正分离, 显然 是因为如此大的两个个体不可能同时占据一个小地 
方。

与其它研究两物种的空间分离方法相比, $N \times$ $N$ 最近邻体列联表的截表法方便、快捷，具有一定 的可信度, 特别适用于多物种群落。研究过程中发 现, 物种个体数越多, 这个方法就越灵敏; 个体数太 少, 其最近邻体也少, 不能反映真实的分离情况。这 也是本研究最后结果仅涉及个体数大于 10 的树种 的原因。虽然结果仅列出 41 个树种, 但取样和邻体 分析时均涉及了样地中所有树种。Pielou (1961) 所 研究的样地中刚好只有两种树:西黄松 (Pinus ponderosa) 和花旗松 (Pseudotsuga menzlesii) , 不存在别的 树种的干扰。对于多物种的群落如热带雨林, 如果 仅选择其中两种作 $2 \times 2$ 最近邻体列联表分析, 则无 法准确反映两物种的真实分离, 因为它们的最近邻 体位置可能为其它物种占据。所以，我们引入了 $N$ $\times N$ 最近邻体列联表。与 Pielou(1961)一样, 我们的 基株包括了样地中所有个体, 真实地反映了整个样 地树种的种间空间分离关系。然而, 如何确定样地 中如此众多的基株-最近邻体种对及其距离, 是一项 浩大的工作 其应用过去一直受到限制, 而利用 GIS 及其邻体分析扩展模块, 我们可以根据植株坐标来 方便快捷地计算得出大样地中的这些种对, 使最近 邻体法的普遍使用成为可能。但是，如何解决 $N \times$ $N$ 列联表截表的统计检验，仍是一个有待统计学家 和生态学家共同努力的问题。

\section{参 考 文 献}

Bawa, K. S. \& P. A. Opler. 1977. Spatial relationship between staminate and pistillate plants of dioecious tropic forest trees. Evolution, 31: $64 \sim 68$.

Clark, P. J. \& F. C. Evans. 1954. Distance to nearest neighbour as a measure of spatial relationships in populations. Ecology, 35: $445 \sim 453$.

Coomes, D. A., M. Rees \& L. Turnbull. 1999. Identifying aggregation and association in fully mapped spatial data. Ecology, 80: $554 \sim 565$.

de Jong, P., L. W. Aarssen \& R. Turkington. 1983. The use of contact sampling in studies of association in vegetation. Journal of Ecology, 71: $545 \sim 559$.

Diggle, P. J. 1983. Statistical analysis of spatial point patterns. London: Academic Press. $1 \sim 148$.

Dixon, P. 1994. Testing spatial segregation using a nearest-neighbor contingency table. Ecology, 75: $1940 \sim 1948$.

Füldner, K. 1995. Zur Strukturbeschreibung in Mischbeständen. Forstarchiv, 66: $235 \sim 240$.
Greig-Smith, P. 1983. Quantitative plant ecology. 3rd ed. Oxford: Blackwell Scientific Publications. 19 128.

Hamill, D. N. \& S. J. Wright. 1986. Testing the dispersion of juveniles relative to adults: a new analytic method. Ecology, 67: $952 \sim 957$.

Kint, V., N. Lust, R. Ferris \& A. F. M. Olsthoorn. 2000. Quantification of forest stand structure applied to Scots pine ( $\mathrm{Pi}$ nus sylvestris L.) forests. Investigación Agraria: Sistemsy Recursos Forestales, 1: $147 \sim 164$.

Law, R., A. Mclellan \& A. Mahdi. 1993. Spatio-temporal processes in a calcareous grassland. Plant Species Biology, 8: 175 $\sim 193$.

Lian, J. Y. (练琚口) \& S. X. Yu(余世孝). 2001. Floristic characters of Formation Dacrydium pierrei-Syzygium araiocladum in the tropical montane rain forest at Bawangling Nature Reserve, Hainan Island. Journal of Tropical and Subtropical Botany (热带 亚热带植物学报), 9:101 107. (in Chinese with English abstract)

Pielou, E. C. 1961. Segregation and symmetry in two-species populations as studied by nearest neighbour relations. Journal of Ecology, 49: $255 \sim 269$.

Pielou, E. C. 1969. An introduction to mathematical ecology. New York: John Wiley \& Sons. 82 212.

Pielou, E. C. 1977. Mathematical ecology. New York: John Wiley \& Sons. $1 \sim 385$.

Reich, R. M. \& R. Davis. 1998. Quantitative spatial analysis. Fort Collins: Colorado State University. $1 \sim 420$.

Ripley, B. D. 1981. Spatial statistics. New York: Wiley.

Wang, B. S. (王伯荪) , S. X. Yu (余世孝), S. L. Peng (彭 少麟) \& M. G. Li (李鸣光). 1996. Experimental manual of plant coenology. Guangzhou: Guangdong Higher Education Press. (in Chinese)

Whipple, S. A. 1980. Population dispersion patterns of trees in a southern Louisiana hardwood forest. Bulletin of the Torrey Botanical Club, 107: $71 \sim 76$.

Wratten, S. D. \& G. L. A. Fry. 1980. Field and laboratory exercises in ecology. London: Edward Arnold. 56 59.

Yu, S.X. (余世孝), G. W. Zong(宗国威)，Z. Y. Chen(陈兆 莹), R. G. Zang(藏润国) \& Y. C. Yang(杨彦承). 1998. Comparison of ecological entropy with random and systematic sampling. Acta Phytoecologica Sinica (植物生态学报), 22:473 480. (in Chinese with English abstract)

Yu, S.X. (余世孝), H. D. Zhang(张宏达) \& B. S. Wang (王 伯荪). 1993. The tropical montane rain forest of Bawangling Nature Reserve, Hainan Island. I. The pPermanent plots and the community types. Ecological Science (生态科学), 12(2) : 13 18. (in Chinese with English abstract)

Yu, S.X. (余世孝)，R.G. Zang (蔵润国) \& Y. X. Jiang (蒋 有绪). 2001a. Spatial analysis of species diversity in the tropical vegetation along the vertical belt at Bawangling Nature Reserve, Hainan Island. Acta Ecologica Sinica (生态学报), 21: $1438 \sim 1443$. (in Chinese with English abstract)

Yu, S.X. (余世孝)，R.G. Zang (蔵润国) \& Y. X. Jiang (蒋 有绪). 2001b. Species richness-abundance relationships in four types of tropical forest on altitudinal gradient at Bawangling Nature Reserve, Hainan. Acta Phytoecologica Sinica (植物生态学 报), 25:291 297. (in Chinese with English abstract) 